\title{
Magnetic and transport properties of diluted granular multilayers
}

\author{
H. G. Silva, ${ }^{1,2, a)}$ H. L. Gomes, ${ }^{1, b)}$ Y. G. Pogorelov, ${ }^{2, c}$ L. M. C. Pereira, ${ }^{2}$ G. N. Kakazei, ${ }^{2,3}$ \\ J. B. Sousa, ${ }^{2}$ J. P. Araújo, ${ }^{2}$ J. F. L. Mariano, ${ }^{4}$ S. Cardoso, ${ }^{5}$ and P. P. Freitas ${ }^{5}$ \\ ${ }^{1}$ Center of Electronic Optoelectronics and Telecommunications (CEOT), Universidade do Algarve, Campus \\ de Gambelas, 8005-139 Faro, Portugal \\ ${ }^{2}$ IFIMUP and IN-Institute of Nanoscience and Nanotechnology, Departamento de Física, Faculdade de \\ Ciências, Universidade do Porto, Rua do Campo Alegre, 687, 4169-007 Porto, Portugal \\ ${ }^{3}$ Institute of Magnetism, National Academy of Sciences of Ukraine, 36 b Vernadskogo Blvd., 03142 Kiev, \\ Ukraine \\ ${ }^{4}$ Departamento de Física, Universidade do Algarve, Campus de Gambelas, 8005-139 Faro, Portugal \\ ${ }^{5}$ INESC-MN and IN-Institute of Nanoscience and Nanotechnology, Rua Alves Redol, 9-1, 1000-029 Lisboa, \\ Portugal
}

(Received 11 June 2009; accepted 28 October 2009; published online 4 December 2009)

The magnetic and transport properties of $\mathrm{Co}_{80} \mathrm{Fe}_{20}(t) / \mathrm{Al}_{2} \mathrm{O}_{3}(4 \mathrm{~nm})$ multilayers with low nominal thicknesses $t=0.7$ and $0.9 \mathrm{~nm}$ of $\mathrm{Co}_{80} \mathrm{Fe}_{20}$ granular layers are studied. Magnetic studies find a superparamagnetic state above the blocking temperature $T_{b}$ (of field-cooled/zero-field-cooled splitting) that grows with $t$ and decreases with $H$. The low-voltage Ohmic tunnel transport passes to non-Ohmic $I \propto V^{3 / 2}$ law for applied fields above $\sim 500 \mathrm{~V} / \mathrm{cm}$. At fixed $V$, the temperature dependence of conductance reveals an anomalous dip around $\sim 220 \mathrm{~K}$, which can be attributed to the effect of surface contamination by supercooled water. Current-in-plane tunnel magnetoresistance (MR) ratio tends, at lower $t$, to higher maximum values ( $\sim 8 \%$ at room temperature) but to lower field sensitivity. This may indicate growing discorrelation effect (e.g., between shrinking areas of correlated moments) in this regime and corroborates the deficit of granule magnetization estimated from the Inoue-Maekawa MR fit, compared to that from direct magnetization measurements. MR displays a mean-field-like critical behavior when $t$ approaches the point of superparamagnetic/ superferromagnetic transition $\left(t_{c} \sim 1.3 \mathrm{~nm}\right.$ at room temperature) from below, different from the formerly reported percolationlike behavior at approaching it from above. With growing temperature, MR reveals, beyond the common decrease, an anomalous plateau from $T_{b} \sim 30-50 \mathrm{~K}$ up to some higher value $T^{*} \sim 150-200 \mathrm{~K}$, not seen at higher $t$. (c) 2009 American Institute of Physics.

[doi:10.1063/1.3266010]

\section{INTRODUCTION}

Intensive research is being done on insulating magnetic granular films during the past decade. Spin-dependent tunneling gives rise to important magneto-electrical properties, as tunnel magnetoresistance (TMR) with high sensitivity to magnetic field and low magnetization coercivity. Therefore, these systems are appealing candidates for next generation high density magnetic memories, low magnetic field sensors, and spin-polarized electrodes in new magnetic tunnel junctions (MTJs). Various granular films have been studied with granules of different ferromagnetic $(\mathrm{FM})$ metals $(\mathrm{Ni}, \mathrm{Co}, \mathrm{Fe}$, $\mathrm{CoFe}$, and $\mathrm{NiFe})$ and distinct insulating hosts $\left(\mathrm{SiO}_{2}, \mathrm{TiO}_{2}\right.$, $\mathrm{ZrO}_{2}, \mathrm{Al}_{2} \mathrm{O}_{3}$, and $\left.\mathrm{MgO}\right) .{ }^{1,2}$ Between them, especially interesting objects are the discontinuous magnetic layers and multilayers, since two-dimensional (2D) geometry favors to more pronounced effects of dipolar coupling between magnetic granules. Experiments on such films show that their magneto-electric properties are mainly controlled by the concentration of magnetic material, or by the nominal thickness $(t)$ of a 2D granular layer (that is its thickness if it were continuous). Presently, the correlation between the transport

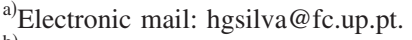

${ }^{b)}$ Electronic mail: hgomes@ualg.pt.

${ }^{c)}$ Electronic mail: ypogorel@fc.up.pt.
}

processes and the film topology (obviously dependent on the nominal thickness of granular layers) is the main objective in all these studies. Three principal transitions are observed with varying concentration (at fixed and high enough temperatures). The first one is that from metallic to activated electrical conduction, controlled by the structural percolation of metallic granules in the insulating matrix, ${ }^{3}$ immediately followed by a second one, that from FM to superferromagnetic (SFM) state. ${ }^{4}$ The third one is that from SFM to superparamagnetic (SPM) [accompanied by the so-called superspin glass (SSG) phase at low enough temperatures $\left.{ }^{5}\right]$. The SFM state is attributed to preferable alignment of magnetic moments on randomly distributed separate granules due to the dipolar coupling between them, controlled by the socalled magnetic percolation. ${ }^{6,7}$ In fact, the SFM-SPM transition [above a certain glass transition temperature $T_{g}$ (Ref. 8)] brings about the most pronounced MR effects, in particular, its sensitivity to low magnetic field. However, up to this moment, certain technical problems prevented more detailed study of granular layers of lower nominal thickness (due to their very high resistivity values) that could clarify magnetotransport mechanisms in these materials.

With this purpose, we study here granular films with low concentration of FM material, below the metal-insulator and SFM-SPM thresholds. This regime, where the system con- 
tains granules of few nanometer size at bigger separation, is usually referred to as "diluted limit." Although the magnetic properties of these systems have been exhaustively studied (see, e.g., Refs. 5 and 9; for a review in supermagnetism the interested reader is referred to Ref. 8), to our knowledge, there is lack of transport and magnetotransport studies in this limit, as compared to that of higher concentration. Therefore, in order to complement the previous works, (magneto)transport and magnetic studies in the diluted limit are presented below. Actually, a recent transport study has found interesting resistive switching effects, ${ }^{10}$ thus reinforcing the demand for a better comprehension of transport phenomena in this limit. We are particularly interested in the relationship between magnetization and TMR (for different temperatures) and how they are affected upon variation of the nominal thickness of granular layer (or the intergranular distance).

Due to the high resistance of the samples the analysis is mainly restricted to high temperatures $(T \gtrsim 100 \mathrm{~K})$, above the glass temperature $T_{g}$, so that a SPM or modified SPM approximation is reasonable. Lacking any precise measurement of $T_{g}$, we roughly estimate it by blocking temperature $T_{b}\left(T_{b} \geq T_{g}\right)$, determined from the splitting of conventionally obtained field-cooled (FC) and zero-field-cooled (ZFC) magnetization curves (see below).

\section{EXPERIMENTAL}

The discontinuous metal-insulator multilayers (DMIMs) under study consist of ten $\mathrm{Co}_{80} \mathrm{Fe}_{20} / \mathrm{Al}_{2} \mathrm{O}_{3}$ bilayers with the last capping $\mathrm{Al}_{2} \mathrm{O}_{3}$ layer. Due to nonwetting of amorphous $\mathrm{Al}_{2} \mathrm{O}_{3}$, thin enough $\mathrm{Co}_{80} \mathrm{Fe}_{20}$ layers get discontinuous, resulting in granular structure. Two particular samples were considered: one with the $\mathrm{Co}_{80} \mathrm{Fe}_{20}$ nominal thickness $t$ $=0.7 \mathrm{~nm}$ and the other with $t=0.9 \mathrm{~nm}$. All the $\mathrm{Al}_{2} \mathrm{O}_{3}$ interlayers have $\approx 4 \mathrm{~nm}$ thickness, except for the $3 \mathrm{~nm}$ capping layer. The diluted films were deposited on glass substrates using Xe-ion beam sputtering. ${ }^{6}$ The formerly done structural studies $^{3,11}$ reveal the presence of well defined spherical magnetic granules. The magnetic measurements with Quantum Design MPMS-5 superconducting quantum interferometry device concerned FC and ZFC magnetizations under $10 \mathrm{Oe}$ in the 5-300 $\mathrm{K}$ temperature range and magnetization versus applied field at different temperatures. Charge transport measurements in the current-in-plane (CIP) geometry, using two gold contacts $(3 \mathrm{~mm}$ long, evaporated on top of films at $100 \mu \mathrm{m}$ separation) and the Keithley 6487 low-current/ high-resistance picoammeter/voltage source, yielded the conductivity versus temperature (at $V=20 \mathrm{~V}$ ) in the 50-300 K temperature range, the current-voltage characteristics, and the MR loops (at $V=20 \mathrm{~V}$ ) in the -9 to $9 \mathrm{kOe}$ field range at different temperatures.

\section{RESULTS AND DISCUSSION}

\section{A. Magnetic properties}

The low-field magnetization curves as a function of temperature, $M(T)$, are shown in Fig. 1(a). They have typical aspect for disordered granular systems, with Curie-Weiss behavior at high temperatures and onset of irreversibility at a blocking temperature $T_{b}$, below which the FC and ZFC
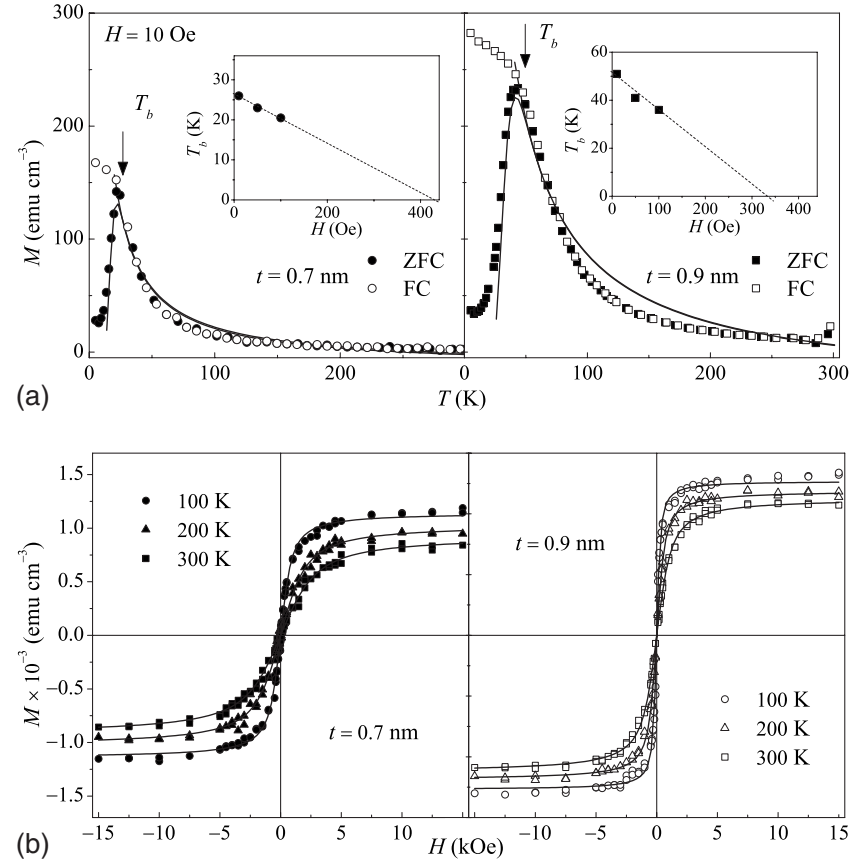

FIG. 1. (a) Magnetization of $\mathrm{Al}_{2} \mathrm{O}_{3}(3.0 \mathrm{~nm}) /\left[\mathrm{Co}_{80} \mathrm{Fe}_{20}(t) /\right.$ $\left.\mathrm{Al}_{2} \mathrm{O}_{3}(4.0 \mathrm{~nm})\right]_{10}$ films with $t=0.7$ and $0.9 \mathrm{~nm}$ under $H=10$ Oe as a function of temperature. The insets show the $T_{b}$ decay with the applied magnetic field. (b) Magnetization vs field cycles at different temperatures.

curves split out. As seen from Fig. 1(a), its values $T_{b} \approx 25$ and $\approx 50 \mathrm{~K}$ for $t=0.7$ and $0.9 \mathrm{~nm}$, respectively, display the expected decrease with decreasing nominal thickness. Also $T_{b}$ somewhat decreases with applied magnetic field [insets in Fig. 1(a)], in agreement with the previous studies for higher $t$ values. ${ }^{12}$ This shift is probably related to a de AlmeidaThouless-type phase boundary of the low-T SSG state. ${ }^{13}$ In the same agreement, the extrapolation of ZFC data versus applied field to $H \rightarrow 0$ (not shown) indicates absence of SFM state even at low temperatures.

Then, fitting essentially the high temperature behavior within the SPM approximation, we can estimate the mean diameter of granules $(d)$, its standard deviation $(s)$, and the effective anisotropy constant $\left(K_{\text {eff }}\right)$. To do this, we solve analytically the model expression suggested by Respaud et al. ${ }^{14}$ for SPM granules with log-normal size distribution (see Appendix). The resulting formulas with the fitting parameters given in Table I provide a fair fit to the experimental data as shown in Fig. 1(a). The values of saturate magnetization $\left(M_{s}\right)$ are in rough agreement with the studies of $\operatorname{Bardos}^{15}$ for bulk CoFe alloys. The inferred average granule diameters of 4.1 and $5.4 \mathrm{~nm}$ are in a fair agreement with the structural analysis on these samples, ${ }^{3,11}$ and the values of mean dispersion $s$ confirm a narrow (quasimonodisperse) distribution.

TABLE I. Fitting parameters for the low-field magnetization curves as a function of temperature, Fig. 1(a).

\begin{tabular}{ccccc}
\hline \hline $\begin{array}{c}t \\
(\mathrm{~nm})\end{array}$ & $\begin{array}{c}M_{s} \\
(\mathrm{G} \mathrm{s})\end{array}$ & $\begin{array}{c}d \\
(\mathrm{~nm})\end{array}$ & $s$ & $\begin{array}{c}K_{\mathrm{eff}} \\
\left(10^{6} \mathrm{erg} \mathrm{cm}^{-3}\right)\end{array}$ \\
\hline 0.7 & 1947 & 4.1 & 0.17 & 1.56 \\
0.9 & 2410 & 5.4 & 0.19 & 1.30 \\
\hline \hline
\end{tabular}


The obtained $K_{\text {eff }}$ values also fairly agree with those reported in literature. ${ }^{16}$ It should be mentioned here that more comprehensive magnetic analyses on these systems should involve a detailed inspection of the SSG state as done in Refs. 5 and 9 , where the aging, memory, and rejuvenation effects ${ }^{8}$ clarify the nature of this state. Nevertheless, as mentioned above, such a treatment is beyond the scope of the present paper, focused instead in the higher temperatures with approximately SPM behavior.

Keeping in mind the previous considerations, the magnetization curves as a function of the applied field $M(H)$ for different temperatures are presented in Fig. 1(b). Their highfield saturation was assured after subtraction of a notable diamagnetic contribution from the glass substrate and amorphous $\mathrm{Al}_{2} \mathrm{O}_{3}$ matrix. The magnetization curves for granular systems are often described in the approximation of two uncoupled subsystems of magnetic granules, large and small sized. ${ }^{5}$ A similar treatment by Zhu et al. ${ }^{17}$ in $\mathrm{Fe}-\mathrm{Al}_{2} \mathrm{O}_{3}$ films considered three uncoupled SPM subsystems (of small, medium, and large granules). Subsequently, Hattink et al. ${ }^{16}$ considered a log-normal distribution of SPM granules. For the present case, reduction in the granule size distribution to a simple monodisperse with resulting expression $M(H)$ $=N \mu L\left(\mu H / k_{\mathrm{B}} T\right)$ was used. Here $N$ is the total number of granules in the sample, $\mu$ is the mean magnetic moment of a granule, $L(x)=\operatorname{coth} x-1 / x$ is the Langevin function, and $k_{\mathrm{B}}$ is the Boltzmann constant. In spite of its simplicity, this approach fits reasonably with the experimental data [see Fig. 1(b)], in agreement with the expected SPM state of diluted systems and the narrow size distribution by the $M(T)$ fit.

\section{B. Charge transport properties}

Current-voltage $(I-V)$ characteristics are generally nonOhmic, as shown in Fig. 2. They are only linear at low voltages, while a $I \propto V^{\gamma}$ power law dominates at electrical fields above $\sim 500 \mathrm{~V} / \mathrm{cm}$. Nonlinear $I-V$ characteristics in diluted granular films have been reported previously by Chayka et al. ${ }^{18}$ They argued this to be a result of impurity mediated conductivity in the insulator $\mathrm{Al}_{2} \mathrm{O}_{3}$ matrix. Actually, nonlinear $I-V$ behavior is typical of charge injection into insulators and has been intensively studied since the Mott and Gurney works ${ }^{19}$ in the context of space-charge-limited currents (SCLCs). That model predicts Ohmic behavior at low voltages followed by a $I \propto V^{2}$ law, for trap-free solids, ${ }^{19,20}$ or by $I \propto V^{1+l}$ with the trap/carrier ratio $l \geq 1$, for trap-limited insulators. $^{20-22}$ Due to its accuracy, SCLC has been largely used to measure charge mobilities and trap characteristic energies in a variety of materials. ${ }^{23}$ However, the SCLC models cannot satisfactorily describe our data since the inferred $\gamma$ values are typically $\approx 3 / 2$, well below the minimum SCLC value of $\gamma=2$ (for the trap-free case). Apparently, this is due to the specifics of tunnel conduction in the considered system, where the space-charge effects are due to extra charges localized on nanometric metallic granules rather than on atomic traps. Besides, the $2 \mathrm{D}$ distribution of granules in a layer favors to faster convergence of Coulomb fields from local charge fluctuations in the average distribution of accumulated charge. The theory, ${ }^{24,25}$ considering planar arrays of
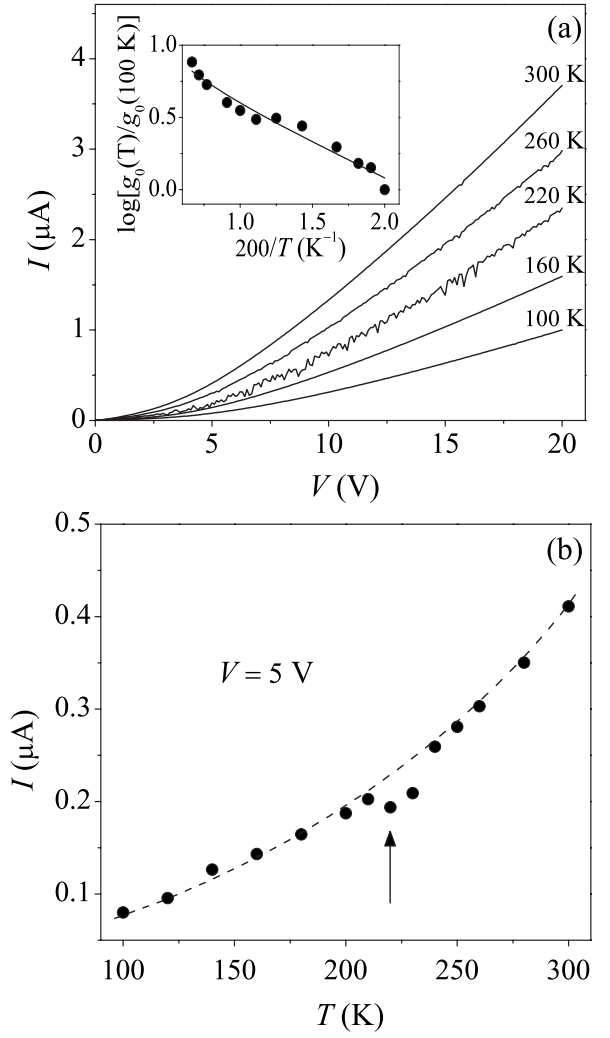

FIG. 2. (a) $I-V$ characteristics for thickness $t=0.9 \mathrm{~nm}$ at different temperatures; Inset: logarithmic representation of the low-voltage Ohmic conductivity, $g_{0}(t)$, divided by $g_{0}(T=100 \mathrm{~K})$ as a function of the inverse temperature. (b) Current vs temperature (with $V=5 \mathrm{~V}$ ) for the same thickness.

single-sized granules adopting single (positive or negative) charges, indeed provides the $I \propto V^{3 / 2}$ law at high enough voltages, while the low-voltage, Ohmic conductivity is predicted to depend on temperature as

$$
g_{0}(T) \propto\left[\left(\frac{T}{T_{1}}+1\right) \frac{2}{2+\exp \left(T_{c} / T\right)}\right]^{1 / 2},
$$

where $T_{c}=e^{2} /\left(2 \varepsilon_{\text {eff }} \bar{d} k_{\mathrm{B}}\right)$ relates to the charging energy for a granule of mean size $\bar{d}$ in the medium with effective dielectric constant $\varepsilon_{\text {eff }}$ and $T_{1} \propto T_{c}$. This formula displays a nonexponential temperature dependence for activated transport in the granular system due to the interplay between temperature dependent number of charge carriers and their mobility. As shown in the inset to Fig. 2(a), our experimental data on $g(T)$ for the $t=0.9 \mathrm{~nm}$ sample are fairly fitted with the choice of $T_{c}=200 \mathrm{~K}$, corresponding to the parameter values $\varepsilon=100$ (high values of the dielectric constant were previously reported ${ }^{24}$ ) and $\bar{d}=5 \mathrm{~nm}$, in a reasonable concordance with the above analysis of magnetization data. Nevertheless, a rather notable deviation of the experimental points from the fitted dependence of $\log \left[g_{0}(T) / g_{0}(100 \mathrm{~K})\right]$ versus $T^{-1}$ is observed in the range $T \sim 200-220 \mathrm{~K}$ (see below).

The temperature dependence of current at a finite, but moderate voltage $(5 \mathrm{~V})$ for the $t=0.9 \mathrm{~nm}$ sample is shown in Fig. 2(b). As expected, the current generally grows with temperature, except for a narrow temperature range around $\sim 220 \mathrm{~K}$, where a clear dip is observed. Such an anomaly, as well as that indicated above in the low-voltage conductivity, 


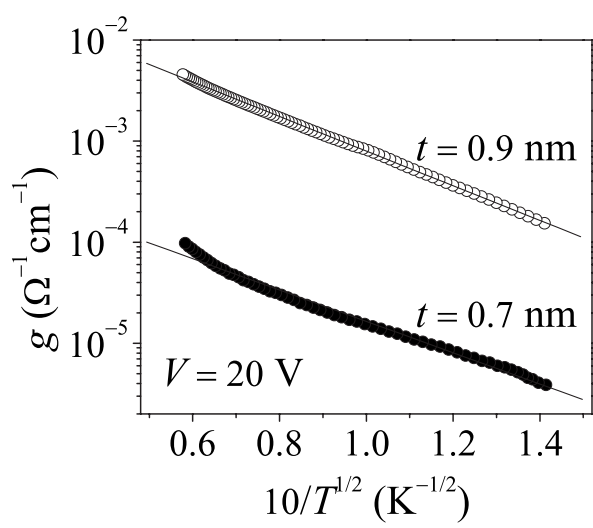

FIG. 3. Conductivity as a function of $T^{-1 / 2}$ for thicknesses $t=0.7,0.9 \mathrm{~nm}$ [fitted with the law of Sheng et al. (Ref. 28)].

can be compared with anomalies reported around 200-220 K in a variety of materials and attributed to a phase transition of unknown origin. ${ }^{26}$ In fact, it was recently shown in organic thin film transistors ${ }^{27}$ that a metastable state of confined water, known as "supercooled water" and persisting near the film surface below the common freezing point down to $T \sim 200-220 \mathrm{~K}$, can cause important carrier trapping. In spite of high vacuum kept during the measurements in our films, there could still remain water contamination and the related carrier trapping could reduce conductance. Carrier trapping by surface water explains qualitatively the observed behavior: there are no water-related traps at low temperatures but, as the temperature of phase transition for surface water is reached, emerging defects can suddenly trap carriers. If the current through the device is low enough, this causes a sizable decrease in the overall current (through device +through surface) until all the traps are filled. Once all the traps are exhausted (filled), the overall current recovers the monotonous behavior by thermal activation. This carrier trapping model is in agreement with the observation that the lower the applied voltage (lower current), the more pronounced is the dip in the temperature dependent curves: The greater part of current has to be spent to fill all the traps generated by the phase transition. Notably, such an effect was not detected previously in granular layers of higher nominal thickness (and higher conductivity) and it could be also responsible for the outburst of noise in the $I-V$ curves at $T \sim 220 \mathrm{~K}$ shown in Fig. 2(a). Similar effects in MR will be discussed in Sec. III C.

To further clarify the charge transport mechanism, current versus temperature measurements were done at yet higher voltages $(V=20 \mathrm{~V})$. For comparison with the literature data, it is suitable to consider again electrical conductivity $g$, rather than current. In Fig. 3 , the plots of $g$ as a function of temperature are shown for the two samples. The straight lines are the fits to the following law of Sheng et al. $:^{28}$

$$
g(T)=g^{(0)}+g^{(1)} \exp \left[-\sqrt{C_{0} /\left(k_{\mathrm{B}} T\right)}\right],
$$

where $g^{(0)}$ is the zero temperature conductivity, $g^{(1)}$ is the temperature independent prefactor, and $C_{0}$ is the tunnel activation energy. The fitting parameters are presented in Table II, and they agree with the literature data. ${ }^{16,18}$ The $C_{0}$ values
TABLE II. Characteristic transport parameters for two nominal thickness values extracted from Fig. 3.

\begin{tabular}{cccc}
\hline \hline $\begin{array}{c}t \\
(\mathrm{~nm})\end{array}$ & $\begin{array}{c}g^{(0)} \\
\left(\Omega^{-1} \mathrm{~cm}^{-1}\right)\end{array}$ & $\begin{array}{c}g^{(1)} \\
\left(\Omega^{-1} \mathrm{~cm}^{-1}\right)\end{array}$ & $\begin{array}{c}C_{0} \\
(\mathrm{meV})\end{array}$ \\
\hline 0.7 & $2.1 \times 10^{-5}$ & $5.9 \times 10^{-4}$ & 27.4 \\
0.9 & $1.3 \times 10^{-3}$ & $4.1 \times 10^{-2}$ & 33.5 \\
\hline \hline
\end{tabular}

are almost the same for both thicknesses and indicate thermally assisted tunneling between neighbor granules, as expected for these disordered diluted systems.

\section{Magnetotransport properties}

In magnetic granular films, charge-carrier tunneling depends on the relative angle $\theta$ between magnetic moments of neighbor granules as $\propto \cos \theta / 2 .^{29}$ In the SPM state at $B \sim 0$, the mean magnetic moments of granules are randomly oriented, producing a significant tunnel resistance. With applied magnetic field, the moments get aligned with the field direction, then decreasing tunnel resistance defines a negative MR. Figure 4(a) shows representative room-temperature MR curves for both thickness values. Notice steeper dependence of $\operatorname{MR}(H)$ at low fields with growing thickness from $t=0.7$ to $0.9 \mathrm{~nm}$. Such decrease in the low-field MR sensitivity on decreasing thickness prevails at all the measured temperatures. In this context, the low-field $\operatorname{MR}(H)$ sensitivity was reported before to reach its sharp maximum at a critical thickness $t^{*}=1.3 \mathrm{~nm}$ (at room temperature) ${ }^{3,30}$ and then to
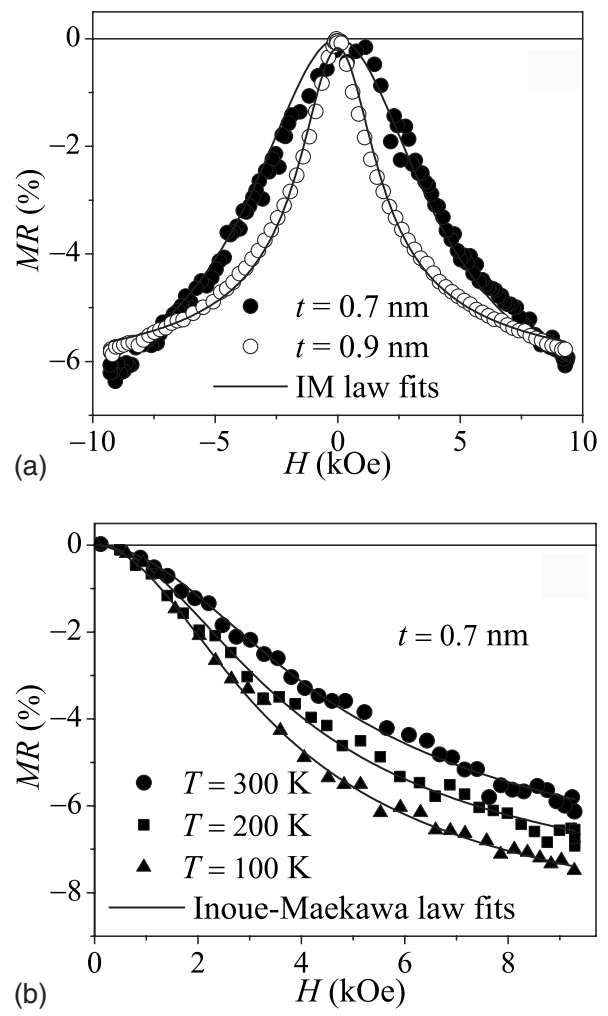

FIG. 4. (a) Field dependence of room-temperature MR at different thicknesses $t=0.7,0.9$, where the IM law for 3D granular films (Ref. 31) is used for fitting MR vs $H$; (b) IM fits at different temperatures for $t=0.7 \mathrm{~nm}$ (similar fits result for $t=0.9 \mathrm{~nm}$ ). 


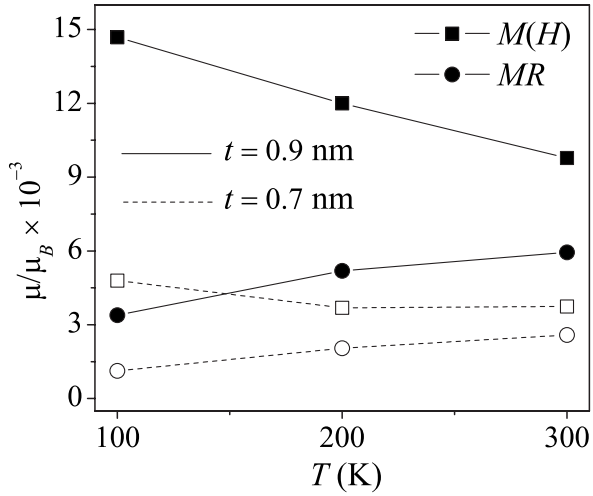

FIG. 5. Comparison between the mean magnetic moments of the granules (in unities of $\mu_{B}$ ) calculated from the fits to the MR (-) and from the magnetization curves $M(H)(\mathbf{\square})$

decrease upon decreasing thickness, mainly due to weakening of magnetic dipolar interactions $\sim 1 / r^{3}$, with increasing intergranule distance $r$. In the SPM state, one can try to fit the MR data with the known Inoue-Maekawa (IM) law for three-dimensional (3D) granular films ${ }^{31} \mathrm{MR}=P^{2} M^{2} /(1$ $\left.+P^{2} M^{2}\right)$, with the ratio $P=\left(D_{\uparrow}-D_{\downarrow}\right) /\left(D_{\uparrow}+D_{\downarrow}\right)$ of densities of states $D_{\uparrow(\downarrow)}$ of majority (minority) Fermi electrons and the magnetization $M$ (again expressed by the Langevin function). Even though the IM law can reasonably fit the MR data [Fig. 4(b)], the found fitting parameters are different from those estimated from the magnetization curves $M(H)$. The respective $\mu / \mu_{B}$ values ( $\mu_{B}$ is the Bohr magneton) inferred from $M(H)$ and MR fits are shown in Fig. 5. This relevant discrepancy shows that the IM formula (derived for a 3D granule distribution) does not fully describe layered systems. In such systems, the in-plane correlations prevail over the interplane ones, imposing an essential correction to this classical law written as

$$
\operatorname{MR}(\theta)=\frac{P^{2}\left(1-\langle\cos \theta\rangle_{\perp}\right)}{1-P^{2}\langle\cos \theta\rangle_{\perp}},
$$

with the average \langle\rangle$_{\perp}$ limited to granules in the same layer. ${ }^{6}$ Unfortunately, we do not have a direct experimental access to this average, but it is expected to be high at RT (due to the in-plane dipolar coupling) and to decrease near and below $T_{b}$ (when a short-range disorder onsets). For this reason, a significant low temperature increase in maximum MR is observed (as shown in Fig. 7). Corrections to the IM model are often necessary to properly fit the experimental data. For instance, Mao et al. ${ }^{32}$ also presented a correction to the IM formula taking into account, phenomenologically, the fielddependent correlation length arising from the interparticle coupling in the SPM state. With such a correction they were able to properly fit the results of Hattink et al. ${ }^{16}$

It is interesting that both thicknesses lead to a similar maximum MR $\sim 6 \%$ with $H \approx 9$ kOe at RT, Fig. 4(a). These values are slightly lower of those observed for the same batch films with $t=1.0 \mathrm{~nm}$ subjected to equal fields. ${ }^{3} \mathrm{In}$ fact, higher values can be deduced from extrapolation of the IM law through saturation field values $(H \approx 50 \mathrm{kOe})$. Doing so the maximum MR values of $\sim 8 \%$ and $\sim 6.5 \%$ were found for the $t=0.7$ and $0.9 \mathrm{~nm}$ samples, respectively. Anyway, our
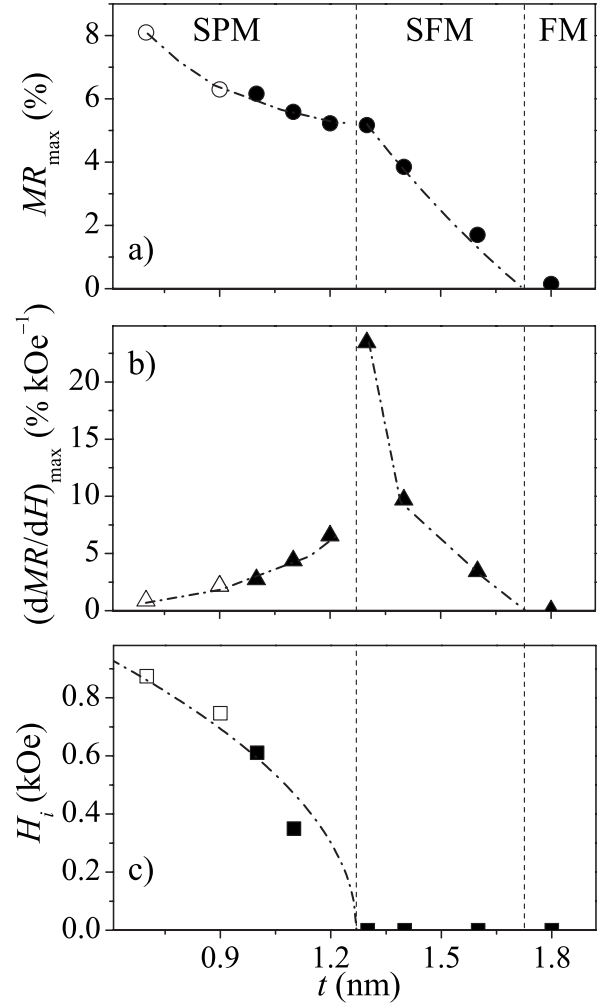

FIG. 6. Critical behavior at the SPM-SFM transition vs nominal thickness $t$ of a granular layer: (a) maximum MR ratio $\left(\mathrm{MR}_{\max }\right)$, (b) MR field sensitivity (dMR/d $H$ ) at room temperature, and (c) field $H_{i}$ of maximum field sensitivity. The solid symbols stand for the previous data (Refs. 12 and 30) and the open symbols are for the present measurements. The vertical lines delimit the ranges of SPM, SFM, and FM phases at this temperature.

ratios are higher than the ones reported by Bručas et al. ${ }^{33}$ studying DMIMs of $\left[\mathrm{Ni}_{81} \mathrm{Fe}_{19}(t \mathrm{~nm}) / \mathrm{Al}_{2} \mathrm{O}_{3}(1.6 \mathrm{~nm})\right]$ type and recently by Garcia-Garcia et al. ${ }^{34}$ considering $[\mathrm{Fe}(t \mathrm{~nm}) / \mathrm{MgO}(3 \mathrm{~nm})]$ multilayers.

Further on, interesting critical behaviors of maximum MR, maximum MR field sensitivity, and field $H_{i}$ of maximum field sensitivity versus $t$ (at RT) are found near the $\mathrm{SPM} / \mathrm{SFM}$ transition $t_{c} \approx 1.3 \mathrm{~nm}$, Fig. 6 . A phase transition occurs at the passage from low to high $t$ displaying a meanfield-like critical behavior when $t$ approaches $t_{c}$ from below (from the SPM side) so that the field $H_{i}$ plays the role of "order parameter," accordingly to a typical law $H_{i}(t)$ $\approx H_{0} \sqrt{t_{c}-t}$, with $H_{0} \approx 1.14 \mathrm{kOe}$. This is different from the formerly reported percolationlike behavior of the SFM order parameter, the spontaneous magnetization $M(H \rightarrow 0)$, at approaching $t_{c}$ from above. ${ }^{12,30}$

Moreover, the temperature dependence of MR is shown in Fig. 7; both thicknesses have anomalous dependencies of $\mathrm{MR}_{\max }$ on temperature. At $T \sim 40 \mathrm{~K}$, a significant enhancement of $\mathrm{MR}_{\max }$ appears, in agreement with the low temperature increase in MR reported in literature. ${ }^{17,35}$ Then, both thicknesses display a tendency to slowly decrease with growing temperature up to $T \sim 160$ and $220 \mathrm{~K}$, for $t=0.7$ and 0.9 $\mathrm{nm}$, respectively. Finally, above $T \sim 220 \mathrm{~K}$, the difference between $\mathrm{MR}_{\max }$ values for the two thicknesses almost disappears, Fig. 7. Interestingly, $\mathrm{MR}_{\max }$ versus $T$ is not properly fitted by the $\mathrm{MR}_{\max } \propto T^{-1}$ law proposed by Helman and Abeles $^{36}$ neither by the $\mathrm{MR}_{\max } \propto \exp \left(-k_{\mathrm{B}} T / E_{m}\right)$ law presented by the model of Mitani et al. ${ }^{35}$ 


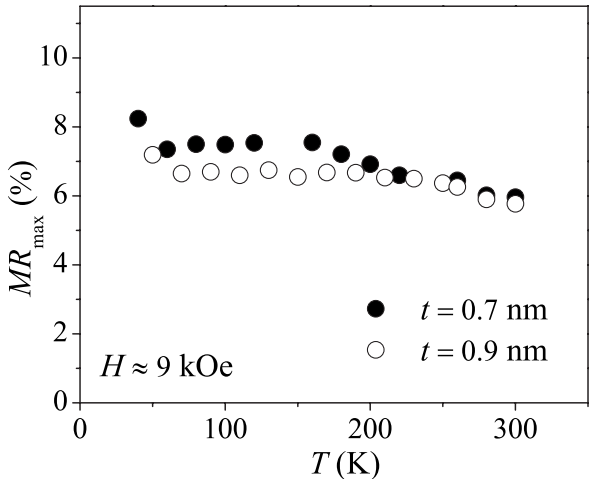

FIG. 7. Maximum MR $(H \approx 9 \mathrm{kOe})$ temperature dependence for both the thicknesses: $t=0.7 \mathrm{~nm}$ and $t=0.9 \mathrm{~nm}$.

Anomalous MR temperature dependencies have been reported previously by Dey et al. ${ }^{37}$ in $\mathrm{La}_{0.7} \mathrm{Ca}_{0.3} \mathrm{MnO}_{3}$ and $\mathrm{La}_{0.67} \mathrm{Sr}_{0.33} \mathrm{MnO}_{3}$ nanoparticles. These authors found that MR is constant up to $T \sim 220 \mathrm{~K}$ and drops sharply after this temperature. Moreover, Bhattacharjee et $a .^{38}$ found in a calorimetric study an anomalous region around $T \sim 200 \mathrm{~K}$, claimed to be due to freezing of the orientational motion of the $\mathrm{H}_{2} \mathrm{O}$ molecules present in the material.

Intriguingly, the temperature dependence of the MR loops for $t=0.9 \mathrm{~nm}$ reveals an anomaly consisting in a sudden change from a noisy behavior with $\mathrm{MR} \propto|H|$ (for $H$ $\rightarrow 0$ ) at $T \leqq 220 \mathrm{~K}$ to a low noise and $\mathrm{MR} \propto H^{2}$ behavior as is shown in Fig. 8. This crossover temperature coincides with the temperature in which the $I-V$ curves become noisy. This suggests that water contamination in our samples indeed causes a degradation of the $I-V$ characteristics due to charge carrier trapping as well as degradation of MR. It is important to mention again that all the measurements were carried out in high vacuum. Even so, it is known that the water absorbed during sample processing and handling is very difficult to be removed.

Finally, the voltage dependence of MR shows no appreciable change for bias voltages from $\sim 0$ up to $\sim 30 \mathrm{~V}$ (not shown). This behavior contrasts with the high-voltage dependence of MR in common MTJs. ${ }^{39}$ Actually, a biasindependent MR has very important technological applications since MR ratios in granular films, although lower than

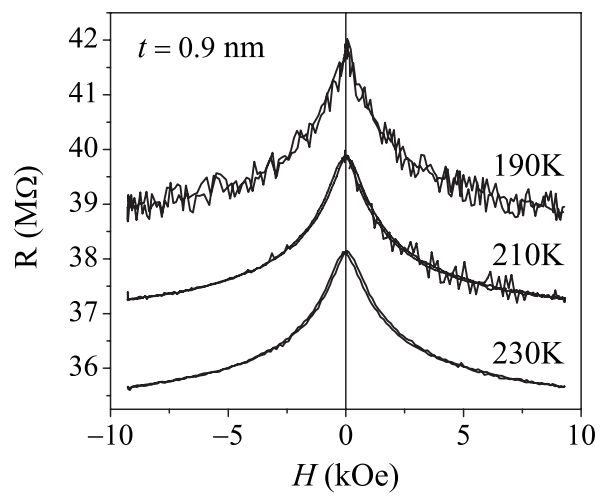

FIG. 8. Sudden change from a noisy MR behavior to a low noise behavior at $T \sim 220 \mathrm{~K}$ for $t=0.9 \mathrm{~nm}$. those in MTJs, can be used in a wider range of bias voltage for a variety of electronic applications and keeping nearly the same MR performance.

\section{CONCLUSIONS}

We have investigated the magnetic and transport properties of diluted granular multilayers, $\mathrm{Co}_{80} \mathrm{Fe}_{20} / \mathrm{Al}_{2} \mathrm{O}_{3}$. The magnetic properties in the superparamagnetic state for sufficiently high temperatures reveal a narrow distribution of small sized granules. The blocking temperature is found to increase with the film nominal thickness. CIP charge transport studies indicate "thermal assisted tunneling," and a reasonable MR ratio at room temperature is found. The study of MR versus nominal thickness of granular layer permitted to detect critical behavior and to establish effective critical parameters of SPM/SFM transition. The importance of in-plane near neighbor granule correlations is evidenced by comparing magnetic and magnetotransport measurements. An unexpected temperature dependence of maximum MR is found, with a significant decrease above a certain temperature (different from the blocking temperature), and unusual noisy behavior either in MR and $I-V$ characteristics was found near this temperature. This anomaly is attributed to water contamination (during processing/handling of samples). Waterrelated traps cause a reduction in the overall device current. This behavior appears suddenly at $\approx 200 \mathrm{~K}$, suggesting its relation to a phase transition of supercooled water.

\section{ACKNOWLEDGMENTS}

The authors acknowledge the support of the Portuguese FCT through Grant Nos. SFRH/BD/24190/2005 (H.G.S.), SFRH/BD/35761/2007 (L.M.C.P.), and "Ciência 2007" program (G.N.K.); and also by the Research Programs: FEDERPOCTI/0155, POCTI/CTM/45252/02, POCTI/CTM/59318/ 2004, and PTDC/FIS/66262/2006. A special acknowledgment is given to Professor J. J. Moura for supplying the electromagnet used in these measurements, and also to J. M. Santos, A. M. Pereira, and D. C. Leitão for fruitful discussions and support.

\section{APPENDIX: ANALYTICAL FORMULAS SUITABLE FOR FITTING ZFC-FC MAGNETIZATION CURVES}

In order to fit the low-field magnetization dependence on temperature, we use the model by Respaud et al., ${ }^{14}$ which presents the total magnetization $M(H)$ as a sum of contributions by SPM and blocked granules in the linear response approximation. The ZFC and FC magnetizations are, respectively, given by the formulas

$$
\begin{aligned}
& M_{\mathrm{ZFC}}(T, H)=M_{s}^{2} H\left(I_{1}+I_{2}\right), \\
& M_{\mathrm{FC}}(T, H)=M_{s}^{2} H\left(I_{1}+\alpha I_{2}\right),
\end{aligned}
$$

where $M_{s}$ is the granule saturated magnetization (taken temperature independent), $\alpha$ is related with the "measurement time" and is very often set as 30 (but not necessarily), and the integrals 


$$
\begin{aligned}
& I_{1}(T)=\frac{1}{3 V k_{\mathrm{B}} T} \int_{0}^{v_{m}(T)} v^{2} f(v) d v, \\
& I_{2}(T)=\frac{1}{3 V K_{\mathrm{eff}}} \int_{v_{m}(T)}^{\infty} v f(v) d v
\end{aligned}
$$

include $V$ the volume of FM materials, and the log-normal distribution function for granule volumes

$$
f(v)=\frac{V}{\sqrt{2 \pi} s \bar{v} v} \exp \left[-\frac{1}{2}\left(\frac{1}{s^{2}} \ln ^{2} \frac{v}{\bar{v}}+s^{2}\right)\right],
$$

with mean value $\bar{v}$ and standard deviation $s$. The integrals (A2) count the contributions by smaller (SPM, $\left.I_{1}\right)$ and bigger (blocked, $I_{2}$ ) granules, distinguished by the characteristic volume $v_{m}=\alpha k_{\mathrm{B}} T / K_{\text {eff }}$, where $K_{\text {eff }}$ is the effective uniaxial anisotropy constant and Brown's theory ${ }^{40}$ parameter [also present in Eq. (A1)] is taken as $\alpha=\ln \left(\tau_{\text {meas }} / \tau_{\text {spin }}\right)$ for given measurement and spin precession times, $\tau_{\text {meas }}$ and $\tau_{\text {spin }}$.

Passing to the variable $x=\ln (v / \bar{v})$ and defining a usual Gaussian structure in the resulting integrands, it can be found that

$$
\begin{aligned}
& I_{1}(T)=\frac{\bar{v}}{6 k_{\mathrm{B}} T} \exp \left(\frac{3 s^{2}}{2}\right)\left[1+\operatorname{erf}\left(\frac{1}{\sqrt{2} s} \ln \frac{v_{m}}{\bar{v}}-\sqrt{2} s\right)\right], \\
& I_{2}(T)=\frac{1}{6 K_{\text {eff }}}\left[1-\operatorname{erf}\left(\frac{1}{\sqrt{2} s} \ln \frac{v_{m}}{\bar{v}}-\frac{s}{\sqrt{2}}\right)\right] .
\end{aligned}
$$

These analytic formulas were used in Eq. (A1) to fit our FC and ZFC magnetization data, as shown in Fig. 1(a).

${ }^{1}$ S. Sankar, D. Dender, J. A. Borchers, D. J. Smith, R. W. Erwin, S. R. Kline, and A. E. Berkowitz, J. Magn. Magn. Mater. 221, 1 (2000).

${ }^{2}$ J. Varalda, W. A. Ortiz, A. J. A. Oliveira, B. Vodungbo, Y.-L. Zheng, D. Demaille, M. Marangolo, and D. H. Mosca, J. Appl. Phys. 101, 014318 (2007).

${ }^{3}$ G. N. Kakazei, Yu. G. Pogorelov, A. M. L. Lopes, J. B. Sousa, P. P. Freitas, S. Cardoso, M. M. Pereira Azevedo, and E. Snoeck, J. Appl. Phys. 90, 4044 (2001).

${ }^{4}$ J. B. Sousa, J. A. M. Santos, R. F. A. Silva, J. M. Teixeira, J. Ventura, J. P. Araújo, P. P. Freitas, S. Cardoso, Yu. G. Pogorelov, G. N. Kakazei, and E. Snoeck, J. Appl. Phys. 96, 3861 (2004).

${ }^{5}$ X. Chen, S. Bedanta, O. Petracic, W. Kleemann, S. Sahoo, S. Cardoso, and P. P. Freitas, Phys. Rev. B 72, 214436 (2005).

${ }^{6}$ G. N. Kakazei, P. P. Freitas, S. Cardoso, A. M. L. Lopes, Yu. G. Pogorelov, J. A. M. Santos, and J. B. Sousa, IEEE Trans. Magn. 35, 2895 (1999) ${ }^{7}$ W. Kleemann, Ch. Binek, O. Petracic, G. N. Kakazei, Yu. G. Pogorelov, M. M. Pereira de Azevedo, J. B. Sousa, and P. P. Freitas, Phys. Rev. B 63, 134423 (2001).

${ }^{8}$ S. Bedanta and W. Kleemann, J. Phys. D: Appl. Phys. 42, 013001 (2009). ${ }^{9}$ S. Sahoo, O. Petracic, W. Kleemann, S. Stappert, G. Dumpich, P. Nordblad, S. Cardoso, and P. P. Freitas, Appl. Phys. Lett. 82, 4116 (2003).

${ }^{10}$ H. Silva, H. L. Gomes, Yu. G. Pogorelov, P. Stallinga, D. M. de Leeuw, J. P. Araújo, J. B. Sousa, S. C. J. Meskers, G. Kakazei, S. Cardoso, and P. P. Freitas, Appl. Phys. Lett. 94, 202107 (2009).
${ }^{11}$ N. A. Lesnik, P. Panissod, G. N. Kakazei, Yu. G. Pogorelov, J. B. Sousa, E. Snoeck, S. Cardoso, P. P. Freitas, and P. E. Wigen, J. Magn. Magn. Mater. 242-245, 943 (2002).

${ }^{12}$ G. N. Kakazei, Yu. G. Pogorelov, J. A. M. Santos, J. B. Sousa, P. P. Freitas, S. Cardoso, N. A. Lesnik, and P. E. Wigen, J. Magn. Magn. Mater. 266, 57 (2003).

${ }^{13}$ S. Sahoo, O. Petracic, Ch. Binek, W. Kleemann, J. B. Sousa, S. Cardoso, and P. P. Freitas, Phys. Rev. B 65, 134406 (2002).

${ }^{14}$ M. Respaud, J. M. Broto, H. Rakoto, A. R. Fert, L. Thomas, B. Barbara, M. Verelst, E. Snoeck, P. Lecante, A. Mosset, J. Osuna, T. Ould Ely, C. Amiens, and B. Chaudret, Phys. Rev. B 57, 2925 (1998).

${ }^{15}$ D. I. Bardos, J. Appl. Phys. 40, 1371 (1969).

${ }^{16}$ B. J. Hattink, M. G. Muro, Z. Konstantinovic, X. Batlle, A. Labarta, and M. Varela, Phys. Rev. B 73, 045418 (2006).

${ }^{17}$ T. Zhu and Y. J. Wang, Phys. Rev. B 60, 11918 (1999).

${ }^{18}$ O. Chayka, L. Kraus, P. Lobotka, V. Sechovsky, T. Kocourek, and M. Jelinek, J. Magn. Magn. Mater. 300, 293 (2006).

${ }^{19}$ N. F. Mott and R. W. Gurney, Electronic Processes in Ionic Crystals (Oxford University Press, New York, 1940).

${ }^{20}$ A. Rose, Phys. Rev. 97, 1538 (1955).

${ }^{21}$ M. Lampert, Phys. Rev. 103, 1648 (1956).

${ }^{22}$ W. Chandra, L. K. Ang, K. L. Pey, and C. M. Ng, Appl. Phys. Lett. 90, 153505 (2007).

${ }^{23}$ C. Tanase, P. W. M. Blom, and D. M. de Leeuw, Phys. Rev. B 70, 193202 (2004).

${ }^{24}$ G. N. Kakazei, A. M. L. Lopes, Yu. G. Pogorelov, J. A. M. Santos, J. B. Santos, P. P. Freitas, S. Cardoso, and E. Snoeck, J. Appl. Phys. 87, 6328 (2000).

${ }^{25}$ J. A. M. Santos, G. N. Kakazei, J. B. Sousa, S. Cardoso, P. P. Freitas, Yu. G. Pogorelov, and E. Snoeck, J. Magn. Magn. Mater. 242-245, 485 (2002).

${ }^{26}$ X. H. Chen, T. F. Li, R. P. Wang, Y. M. Qiu, K. Q. Ruan, L. Z. Cao, L. Shi, Y. B. Jia, G. E. Zhou, and Y. H. Zhang, J. Phys.: Condens. Matter 9, L75 (1997); K. Fujishiro and Y. Uesu, ibid. 8, 6435 (1996); R. Jin, J. He, J. R. Thompson, M. F. Chisholm, B. C. Sales, and D. Mandrus, ibid. 14, L117 (2002).

${ }^{27}$ H. L. Gomes, P. Stallinga, M. Cölle, D. M. de Leeuw, and F. Biscarini, Appl. Phys. Lett. 88, 082101 (2006); H. L. Gomes, P. Stallinga, M. Cölle, F. Biscarini, and D. M. de Leeuw, J. Non-Cryst. Solids 352, 1761 (2006).

${ }^{28}$ P. Sheng, B. Abeles, and Y. Aire, Phys. Rev. Lett. 31, 44 (1973).

${ }^{29}$ J. I. Gittleman, Y. Goldstein, and S. Bozowsky, Phys. Rev. B 5, 3609 (1972).

${ }^{30}$ J. B. Sousa, G. N. Kakazei, Yu. G. Pogorelov, J. A. M. Santos, O. Petracic, W. Kleemann, Ch. Binek, S. Cardoso, P. P. Freitas, M. M. Pereira Azevedo, N. A. Lesnik, M. Rokhlin, and P. E. Wigen, IEEE Trans. Magn. 37, 2200 (2001).

${ }^{31}$ J. Inoue and S. Maekawa, Phys. Rev. B 53, R11927 (1996).

${ }^{32}$ Z. Mao, D. Chen, and Z. He, J. Magn. Magn. Mater. 320, 642 (2008).

${ }^{33}$ R. Bručas, M. Hanson, R. Gunnarsson, E. Wahlström, M. van Kampen, B. Hjörvarsson, H. Lidbaum, and K. Leifer, J. Appl. Phys. 101, 073907 (2007).

${ }^{34}$ A. García-García, A. Vovk, J. A. Pardo, P. Štrichovanec, C. Magén, E. Snoeck, P. A. Algarabel, J. M. De Teresa, L. Morellón, and M. R. Ibarra, J. Appl. Phys. 105, 063909 (2009).

${ }^{35}$ S. Mitani, S. Takanashi, K. Yakushiji, S. Maekawa, and H. Fujimori, Phys. Rev. Lett. 81, 2799 (1998).

${ }^{36}$ J. S. Helman and B. Abeles, Phys. Rev. Lett. 37, 1429 (1976).

${ }^{37}$ P. Dey, T. K. Natha, U. Kumar, and P. K. Mukhopadhyay, J. Appl. Phys. 98, 014306 (2005); P. Dey and T. K. Nath, Phys. Rev. B 73, 214425 (2006).

${ }^{38}$ A. Bhattacharjee, S. Saha, S. Koner, and Y. Miyazaki, J. Magn. Magn. Mater. 312, 435 (2007).

${ }^{39}$ J. S. Moodera, L. R. Kinder, T. M. Wong, and R. Meservey, Phys. Rev. Lett. 74, 3273 (1995).

${ }^{40}$ W. F. Brown, Jr., Phys. Rev. 130, 1677 (1963). 